\title{
Random diffusion and leverage effect in financial markets
}

\author{
Josep Perelló* and Jaume Masoliver ${ }^{\dagger}$ \\ Departament de Física Fonamental, Universitat de Barcelona, Diagonal 647, E-08028 Barcelona, Spain
}

(Received 11 February 2002; published 25 March 2003)

\begin{abstract}
We prove that Brownian market models with random diffusion coefficients provide an exact measure of the leverage effect [J-P. Bouchaud et al., Phys. Rev. Lett. 87, 228701 (2001)]. This empirical fact asserts that past returns are anticorrelated with future diffusion coefficient. Several models with random diffusion have been suggested but without a quantitative study of the leverage effect. Our analysis lets us to fully estimate all parameters involved and allows a deeper study of correlated random diffusion models that may have practical implications for many aspects of financial markets.
\end{abstract}

DOI: 10.1103/PhysRevE.67.037102

PACS number(s): 89.65.Gh, 02.50.Le, 05.40.Jc, 05.45.Tp

The dynamics of particles in random media can be used for a large variety of phenomena in statistical physics and condensed matter [1]. This class of models has been applied to polymer transport, electrospin dynamics of polarons, protein dynamics, and flux lines in high $T_{c}$ superconductors $[1,2]$. Likewise, in modeling financial markets there also exist several approaches based on random diffusion although in mathematical finance these are known as stochastic volatility (SV) models $[3,4]$. The aim of this paper is to stress the importance of the random diffusion approach in market dynamics by explaining the leverage effect, an old phenomenon only very recently quantified [5]. In the past decade, there has been an increasing interest in applying the methods of statistical physics to the study of speculative markets [6]. The present work adopts the same philosophy.

The multiplicative diffusion process known as the geometric Brownian motion (GBM) has been widely accepted as a universal model for speculative markets. The model, suggested by Bachelier in 1900 as an ordinary random walk and redefined in its final version by Osborne in 1959 [7], presupposes a constant "volatility" $\sigma$, that is to say, a constant diffusion coefficient $D=\sigma^{2}$. However, and especially after the 1987 crash, there seems to be empirical evidence, embodied in the so-called "stylized facts," that the assumption of constant volatility does not properly account for important features of markets [8]. It is not a deterministic function of time either (as might be inferred by the evidence of nonstationarity in financial time series) but a random variable. In its more general form one therefore assumes that the volatility $\sigma$ is a given function of a random process $Y(t)$, i.e., $\sigma(t)=\phi(Y(t))$.

We may make an analogy from physics saying that speculative prices $S(t)$ evolve in a random medium determined by a random diffusion coefficient. The randomness of the medium constrains the amplitude of price changes. It is commonly asserted that this amplitude is directly related to the market activity and the number of contracts negotiated. Hence, periods with high market activity indicate large variety of trading positions and this, in turn, implies a considerable dispersion of possible changes in prices at every time

\footnotetext{
*Email address: perello@ffn.ub.es

†Email address: jaume@ffn.ub.es
}

step. In the opposite case, low market activity indicates a small variety of market positions and finally, a small dispersion in possible future prices. In this sense, we may say that market stochastic dynamics fluctuates with an amplitude quantified by a random diffusion coefficient $D$. This coefficient is related to market activity in the same manner as in physics the diffusion coefficient is related to temperature [9]. In this way, we are thus assuming that market activity is not constant but stochastic and is governed by the random arrival of information to the markets.

Most stochastic volatility models studied to date assume that $Y(t)$ is itself a diffusion process that may or may not be correlated with price and these generally differ from each other in the form of the function $\phi[3,4]$. The hypothesis of stochastic volatility was originally suggested to explain the so-called "smile effect" which is related to implicit volatilities in option prices. The smile effect has been thoroughly studied both qualitatively and quantitatively [10,11]. However, other features referred more directly to the statistical properties of SV models are solely studied from a qualitative point of view [12] or by giving numerical coefficients based on ARCH-GARCH models [13].

Our main objective is to prove that leverage can be quantitatively explained in terms of a wide class of correlated SV models. This will allow us to overcome the main objection against SV models: the impossibility of fitting all parameters appearing in these models [4,12]. This, then opens the door to further statistical studies based on SV models for real markets with far-reaching practical consequences for option pricing and risk management.

The leverage effect has its origin in the observation that volatility seems to be negatively correlated with stock returns. These are defined by $R(t)=\ln [S(t) / S(0)]$ where $S(t)$ is the speculative price at time $t$. The first explanation for this empirical fact was suggested by Black [14] and Christie [15]. They say that negative returns increase financial leverage, which extends the risk of the company, and therefore its volatility. Hence the name of "leverage effect." Nevertheless, the cause of this effect is still unclear since another contrary explanation can be given, namely, that an increase of volatility makes the stock riskier which produces a fall of demand. As a result the price of the stock drops [3].

In a very recent paper, Bouchaud et al. [5] performed an empirical study of the leverage effect for both individual 
stocks and indices based on daily data. The volatility-return (negative) correlation was clearly shown to have a definite direction in time - a very confusing fact in the literaturesince correlations have been shown to exist between future volatilities and past returns. Bouchaud et al. conclusively prove from data that the negative correlation decays exponentially in time, and is faster for indices than for individual stocks [5].

In this paper, we present a theoretical study of these correlations and show that a wide class of random diffusion models can be invoked to explain the leverage effect in agreement with experimental observations. The starting point is the GBM model:

$$
d R=\mu d t+\sigma(t) d W_{1}(t),
$$

where $\mu$ is the drift and $\sigma(t)=\sigma(Y(t))$ is a random volatility and $Y(t)$ is a diffusion process,

$$
d Y=f(Y) d t+g(Y) d W_{2}(t) .
$$

In these equations, $W_{i}(t)(i=1,2)$ are Wiener processes, i.e., $d W_{i}(t)=\xi_{i}(t) d t$ where $\xi_{i}(t)$ are zero-mean Gaussian white noises with $\left\langle\xi_{i}(t) \xi_{i}\left(t^{\prime}\right)\right\rangle=\delta\left(t-t^{\prime}\right)$ and only one nonzero cross correlation given by

$$
\left\langle\xi_{1}(t) \xi_{2}\left(t^{\prime}\right)\right\rangle=\rho \delta\left(t-t^{\prime}\right)
$$

$(-1 \leqslant \rho \leqslant 1)$. As is common in finance, Eqs. (1) and (2) are interpreted in the sense of Itô and for the rest of the paper we will follow the Itô convention [16].

Bouchaud et al. [5] quantify the leverage effect by means of a leverage correlation function defined by

$$
\mathcal{L}(\tau) \equiv \frac{1}{Z}\left\langle d X(t+\tau)^{2} d X(t)\right\rangle,
$$

where $X(t) \equiv R(t)-\mu t$ is the zero-mean return and $Z$ $=\left\langle d X(t)^{2}\right\rangle^{2}$ is a convenient normalization coefficient. They have analyzed a large amount of daily relative changes for both market indices and stock share prices finding that

$$
\mathcal{L}(\tau)= \begin{cases}-A e^{-b \tau} & \text { if } \tau>0, \\ 0 & \text { if } \tau<0\end{cases}
$$

$(A, b>0)$ [5]. Hence, there is an exponentially decaying negative correlation between future volatility and past returns changes. No correlation is found between past volatility and future price changes. In this way, they provide a sort of causality to the leverage effect which, to our knowledge, has never been previously mentioned in the literature $[3,4]$.

Let us sketch how correlated SV models are able to exactly reproduce this result. We take Eq. (1) in terms of the zero-mean return and obtain

$$
\mathcal{L}(\tau)=\left\langle\sigma(t) d W_{1}(t) \sigma(t+\tau)^{2} d W_{1}(t+\tau)^{2}\right\rangle / Z .
$$

Note that when $\tau<0$, Itô's rules tell us that $d W_{1}(t)$ is uncorrelated with the remaining random variables. Then, recalling that $\left\langle d W_{1}(t)\right\rangle=0$, we have $\mathcal{L}(\tau)=0$ if $\tau<0$. On the other hand, when $\tau>0, d W_{1}(t+\tau)$ is uncorrelated with the remaining terms and, since $\left\langle d W_{1}(t+\tau)^{2}\right\rangle=d t$, we conclude that

$$
\mathcal{L}(\tau)=\theta(\tau)\left\langle\sigma(t) d W_{1}(t) \sigma(t+\tau)^{2}\right\rangle d t / Z,
$$

where $\theta(\tau)$ is the Heaviside step function and $Z$ $=\left\langle\sigma^{2}(t)\right\rangle^{2} d t^{2}$. Note that we have proved the existence of correlations between future volatilities and past returns but not vice versa. Note also that this is independent of the underlying volatility process $Y(t)$ and of the specific form of $\sigma$ in terms of $Y$.

Suppose now that $Y(t)$ is a diffusion process given by Eq. (2). As is well known, any pair of correlated Wiener process, such as $W_{1}(t)$ and $W_{2}(t)$, satisfies the identity $d W_{1}(t)$ $=\rho d W_{2}(t)+\sqrt{1-\rho^{2}} d W(t)$, where $W(t)$ is a Wiener process independent of $W_{2}(t)$ [so that $W(t)$ is independent of $\sigma]$. Substituting this identity into Eq. (6), we get $\mathcal{L}(\tau)$ in terms of the average $\left\langle\sigma(t) \sigma(t+\tau)^{2} \xi_{2}(t)\right\rangle$. This average can be calculated by means of Novikov's theorem [17] with the result [18]

$$
\mathcal{L}(\tau)=\frac{2 \rho \theta(\tau)}{\left\langle\sigma^{2}(t)\right\rangle^{2}}\left\langle\sigma(t) \sigma(t+\tau) \sigma^{\prime}(t+\tau) \frac{\delta Y(t+\tau)}{\delta \xi_{2}(t)}\right\rangle,
$$

where $\sigma^{\prime}=\partial \sigma(Y) / \partial Y$ and $\delta Y(t+\tau) / \delta \xi_{2}(t)$ is the functional derivative of $Y\left(t+\tau,\left[\xi_{2}\right]\right)$ with respect to $\xi_{2}(t)$ [17].

There is a wide consensus that volatility has the property of reverting to the mean. In other words, there exists a normal level [19] to which the volatility eventually returns [13]. For a general SV model such as Eqs. (1) and (2), the existence of mean reversion implies restrictions on the form of the drift coefficient $f(Y)$. A simple way to incorporate this experimental fact into the model is to assume that $f(Y)$ is linear. That is,

$$
\dot{Y}=-\alpha(Y-m)+g(Y) \xi_{2}(t),
$$

where $\alpha>0$. The formal solution to this equation in the stationary state reads

$$
Y(t)=m+\int_{-\infty}^{t} e^{-\alpha\left(t-t^{\prime}\right)} g\left(Y\left(t^{\prime}\right)\right) \xi_{2}\left(t^{\prime}\right) d t^{\prime},
$$

from which we get [18]

$$
\frac{\delta Y(t+\tau)}{\delta \xi_{2}(t)}=\theta(\tau) e^{-\alpha \tau} g(Y(t)) \exp \left[\int_{t}^{t+\tau} g^{\prime}(Y(s)) \xi_{2}(s) d s\right] .
$$

A substitution of this expression into Eq. (7) yields

$$
\mathcal{L}(\tau)=\rho \theta(\tau) B(\tau) e^{-\alpha \tau},
$$

where

$$
B(\tau)=\frac{2\left\langle\sigma(t) \sigma(t+\tau) \sigma^{\prime}(t+\tau) G(t, t+\tau)\right\rangle}{\left\langle\sigma^{2}(t)\right\rangle^{2}},
$$




$$
G(t, t+\tau)=g(Y(t)) \exp \left[\int_{t}^{t+\tau} g^{\prime}(Y(s)) \xi_{2}(s) d s\right]
$$

In consequence, any SV model of the form given by Eqs. (1) and (8) in which the function $B(\tau)$ increases no faster than $e^{\alpha \tau}$ as $\tau \rightarrow \infty$, satisfies an exponentially decaying leverage as expressed by Eq. (9). Moreover, if $\sigma(Y)$ is an increasing function of $Y$ with fixed sign and $g(Y)$ is positive definite (or $\sigma$ is decreasing and $g$ is negative), we see from Eq. (9) that the correlation coefficient $\rho$ must be negative and driving noises $W_{1}(t)$ and $W_{2}(t)$ are anticorrelated. Equations (9)(11) constitute the main result of the paper.

The exact form of $\mathcal{L}(\tau)$, will depend on the expression of $B(\tau)$ which in turn will depend on the SV model chosen. Within diffusion theory, as is the case of Eq. (8), there are basically three different SV models [4]: (1) The OrnsteinUhlenbeck (OU) model where $\sigma=Y$ and $g(Y)=k$ (a positive constant) [20,21], (2) the exponential Ornstein-Uhlenbeck (expOU) model where $\sigma=e^{Y}$ and $g(Y)=k$ [12], and (3) the Cox-Ingersoll-Ross (CIR) model, also called Heston model [11], where $\sigma=\sqrt{Y}$ and $g(Y)=k \sqrt{Y}$. For all these models the leverage function has the form given by Eq. (9). In the OU model and in the CIR model (the latter with zero-mean reversion, i.e., $\left.m=k^{2} / 4 \alpha\right)$, the leverage function $\mathcal{L}(\tau)$ is, respectively, given by [18]

$$
\begin{gathered}
\mathcal{L}_{1}(\tau)=2 k \rho \theta(\tau)\left[\frac{m^{2}+\left(k^{2} / 2 \alpha\right) e^{-\alpha \tau}}{\left(m^{2}+k^{2} / 2 \alpha\right)^{2}}\right] e^{-\alpha \tau}, \\
\mathcal{L}_{3}(\tau)=4 \frac{\rho \alpha}{k} \theta(\tau) e^{-\alpha \tau},
\end{gathered}
$$

while for the expOU model we have [18]

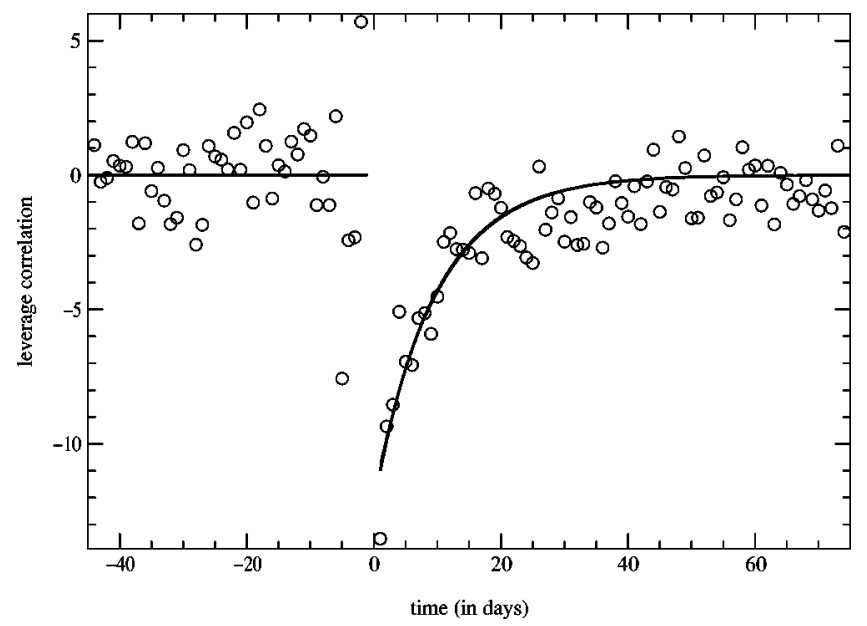

FIG. 1. The leverage effect in the Dow-Jones daily index. We plot the leverage function $\mathcal{L}(\tau)$ for the DJ from 1900 until 2000. We see that there exists a non-negligible correlation when $\tau>0$ and negligible when $\tau<0$ and that correlation strongly fluctuates when $-3<\tau<2$. We also plot a fit with the OU SV leverage function (12) allowing us to estimate $\alpha$ and $\rho$.

$$
\mathcal{L}_{2}(\tau)=2 \rho k \theta(\tau) \exp \left[-m+k^{2}\left(e^{-\alpha \tau}-3 / 4\right) / \alpha\right] e^{-\alpha \tau}
$$

We note that all these models have similar properties and present an exponential decaying leverage as shown in data (see Fig. 1). Nonetheless, CIR and expOU models assume a positive $\sigma$ while for the OU model there is a nonzero, but small, probability of having negative values of $\sigma$ [21].

Any market model, besides being able to reproduce the market dynamics, must provide a systematic way of evaluating its parameters. Almost all current SV models have four parameters to estimate: $\rho, k, m$, and $\alpha$. Until very recently, all existent works on SV models are only able to evaluate two of them. Thus, for instance, Fouque et al. [12] estimate $k$ and $m$ from the empirical second and fourth moment of daily data but cannot estimate $\alpha$ and $\rho$. This is a significant objection to SV models. The situation changes completely when the leverage is measured. Indeed, $k$ and $m$ are obtained, as usual, from the empirical second and fourth moment. Next, by adjusting $e^{-\alpha \tau}$ to the leverage empirical data we are able to estimate $\alpha$. Moreover, comparing the theoretical and empirical leverage at $\tau=0^{+}, \mathcal{L}\left(0^{+}\right)$, we finally obtain $\rho$.
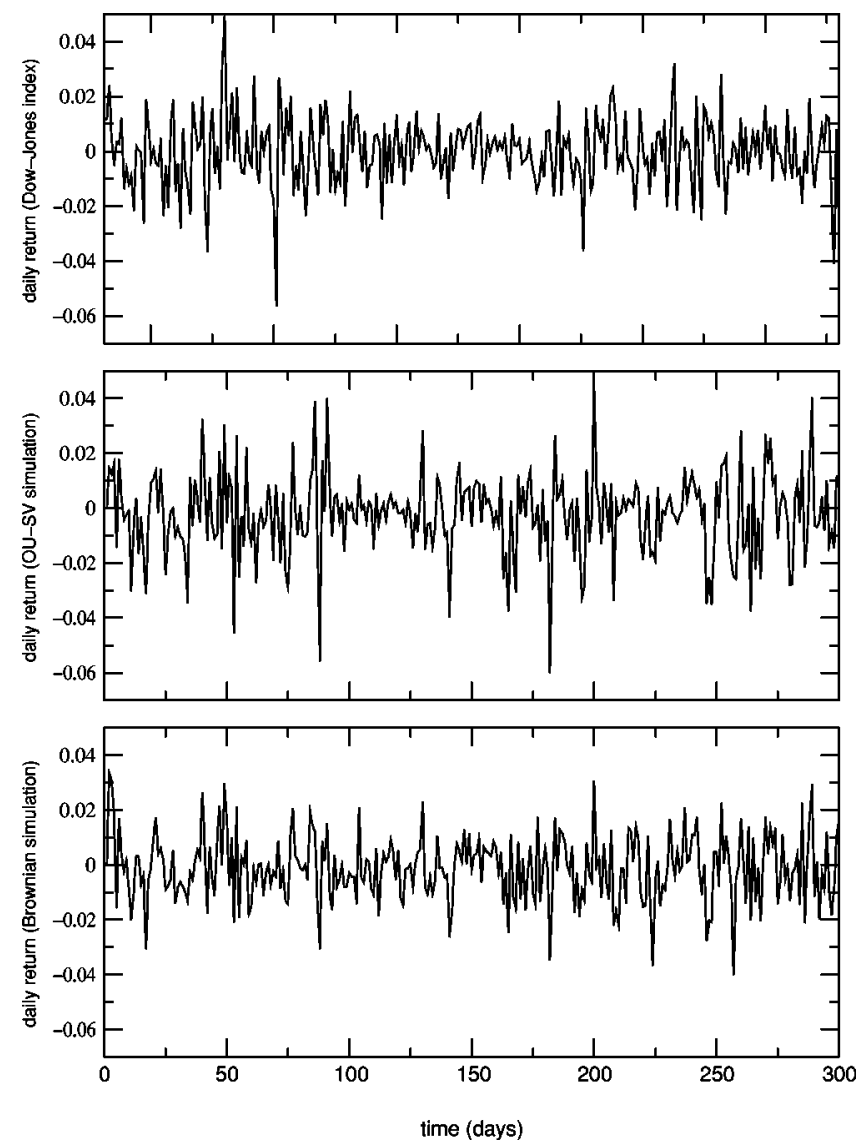

FIG. 2. Dow-Jones index and path simulations. We show a DJ daily returns sample path (top), its simulation by means of the OU SV process (middle), and the GBM simulation (bottom). All parameters of the simulations are estimated from the whole DJ historical time series (1900-2000). Dynamics is traced over 300 days and, for the DJ path, this nearly corresponds to 1999. 
To our knowledge, the only alternative to this method for estimating parameters is given by the recent work of Dragulescu and Yakovenko [22], who study the probability distribution of the CIR model and obtain the parameters by minimizing the mean square deviation between the logarithms of the theoretical and empirical return distributions [22].

Following our procedure, for the Dow-Jones (DJ) daily index and using the OU model, we have estimated $k=(2$ $\pm 1) \times 10^{-3}$ day $^{-1}, m=(16 \pm 5) \% \mathrm{yr}^{-1 / 2}, 1 / \alpha=14 \pm 6$ days, and $\rho=-0.5 \pm 0.1$. Errors are evaluated by considering sample data of 100 years in ten samples of 10 years. From the parameter estimation in every interval, we can obtain a mean and a standard deviation for each parameter of the model.

As an illustration we have simulated, using Eqs. (1) and (8), the OU resulting process with our earlier parameter's estimates. We follow the random dynamics of the daily changes of the zero-mean return, $X(t)$, and compare it with the empirical DJ time series during approximately one trading year. We have also simulated the GBM assuming a constant volatility $\sigma$ whose value is directly estimated from DJ for one century data. These results are shown in Fig. 2. Observe that GBM cannot describe either the largest or the smallest fluctuations of daily returns. We nonetheless see in the figure that the SV model chosen describes periods of high volatility together with periods of very low volatility, resulting in a more similar trajectory to the DJ index than that of the GBM. This is quite remarkable, because we have simulated last year trajectory using the past 100 years of data available of the DJ index thus showing the stability of parameters. However, we also admit that the OU model has a volatility correlation with a single exponential time decay and this is in contradiction with some empirical observations showing a power law decay or, at least, a multiple time scale $[8,21]$. Our present investigations go to this direction [18].

These results suggest further study of statistical properties of random diffusion models with leverage. Several models have been discussed in the literature without being able to discern which is the more realistic one. Now, thanks to the leverage correlation, one can estimate all of the parameters. This facilitates a comparison of different models and empirical statistical properties of markets. Finally, a better knowledge of SV models has nontrivial consequences on option pricing (since the classical Black-Scholes method can be incorporated into the framework of SV models $[10,11]$ ) and, more generally, into risk management strategies.

This work has been supported in part by Dirección General de Investigación under Contract No. BFM2000-0795, and by Generalitat de Catalunya under Contract No. 2000 SGR-00023. We thank M. Bogunya and G. H. Weiss for useful suggestions and a careful reading of the paper.
[1] D. Ben-Avraham and S. Havlin, Diffusion and Reactions in Fractals and Disordered Systems (Cambridge University Press, Cambridge, 2000).

[2] See, e. g, S.B. Santra, W.A. Seitz, and D.J. Klein, Phys. Rev. E 63, 067101 (2001); B. Hess, ibid. 62, 8438 (2000); S.E. Henrickson, M. Misakian, B. Robertson, and J.J. Kasianowicz, Phys. Rev. Lett. 85, 3057 (2000).

[3] E. Ghysels, A.C. Harvey, and E. Renault, in Statistical Methods in Finance, edited by G. S. Mandala and C.R. Rao (NorthHolland, Amsterdam, 1996), Vol. 14.

[4] J.-P. Fouque, G. Papanicolaou, and K.R. Sircar, Derivatives in Financial Markets with Stochastic Volatility (Cambridge University Press, Cambridge, 2000).

[5] J.-P. Bouchaud, A. Matacz, and M. Potters, Phys. Rev. Lett. 87, 228701 (2001).

[6] See, e.g., J.-P. Bouchaud and M. Potters, Theory of Financial Risks (Cambridge University Press, Cambridge, 2000); J. Voit, The Statistical Mechanics of Financial Markets (SpringerVerlag, Berlin, 2001).

[7] The Random Character of Stock Market Prices, edited by P.H. Cootner (MIT Press, Cambridge, MA, 1964).

[8] R. Cont, Quant. Fin. 1, 223 (2001); V. Plerou, P. Gopikrishnan, L.N. Amaral, M. Meyer, and E. Stanley, Phys. Rev. E 60, 6519 (1999).
[9] J.P. Garrahan, E. Moro, and D. Sherrington, Phys. Rev. E 62, R9 (2000).

[10] J.C. Hull and A. White, J. Financ. 42, 281 (1987); L. Scott, J. Fin. Quant Anal. 22, 419 (1987); J. Wiggins, J. Fin. Eco. 19, 351 (1987).

[11] S.L. Heston, Rev. Financ. Stud. 6, 327 (1993).

[12] J-P. Fouque, G. Papanicolaou, and K.R. Sircar, Int. J. Theor. Appl. Finance 3, 101 (2000).

[13] R. Engle and A. Patton, Quant. Fin. 1, 237 (2001).

[14] F. Black, in Proceedings of the 1976 American Statistical Association, Business and Economical Statistics Section (American Statistical Association, Alexandria, VA, 1976), p. 177.

[15] A.A. Christie, J. Fin. Eco. 10, 407 (1982).

[16] J. Perelló, J.M. Porrà, M. Montero, and J. Masoliver, Physica A 278, 260 (2000).

[17] E.A. Novikov, Sov. Phys. JETP 20, 1290 (1965).

[18] J. Perelló, J. Masoliver, and J.-P. Bouchaud, e-print cond-mat/ 0302095.

[19] The normal level of volatility is usually defined by $\lim _{t \rightarrow \infty}\left\langle\sigma^{2}(t)\right\rangle$ (see Ref. [13]).

[20] E.M. Stein and J.C. Stein, Rev. Financ. Stud. 4, 727 (1991).

[21] J. Masoliver and J. Perelló, Int. J. Theor. Appl. Finance 5, 541 (2002).

[22] A. Dragulescu and V. Yakovenko, Quant. Fin. 2, 443 (2002). 